\title{
A!
}

This is an electronic reprint of the original article.

This reprint may differ from the original in pagination and typographic detail.

Siltanen, Samuel; Lokki, Tapio; Kiminki, Sami; Savioja, Lauri

\section{The room acoustic rendering equation}

Published in:

Journal of the Acoustical Society of America

DOI:

$10.1121 / 1.2766781$

Published: 01/01/2007

Document Version

Publisher's PDF, also known as Version of record

Please cite the original version:

Siltanen, S., Lokki, T., Kiminki, S., \& Savioja, L. (2007). The room acoustic rendering equation. Journal of the Acoustical Society of America, 122(3), 1624-1635. https://doi.org/10.1121/1.2766781

This material is protected by copyright and other intellectual property rights, and duplication or sale of all or part of any of the repository collections is not permitted, except that material may be duplicated by you for your research use or educational purposes in electronic or print form. You must obtain permission for any other use. Electronic or print copies may not be offered, whether for sale or otherwise to anyone who is not an authorised user. 


\title{
The room acoustic rendering equation
}

\author{
Samuel Siltanen, ${ }^{\text {a) }}$ Tapio Lokki, Sami Kiminki, and Lauri Savioja \\ Telecommunications Software and Multimedia Laboratory, Helsinki University of Technology, \\ P.O. Box 5400, FIN-02015 HUT, Finland
}

(Received 3 October 2006; revised 4 July 2007; accepted 5 July 2007)

\begin{abstract}
An integral equation generalizing a variety of known geometrical room acoustics modeling algorithms is presented. The formulation of the room acoustic rendering equation is adopted from computer graphics. Based on the room acoustic rendering equation, an acoustic radiance transfer method, which can handle both diffuse and nondiffuse reflections, is derived. In a case study, the method is used to predict several acoustic parameters of a room model. The results are compared to measured data of the actual room and to the results given by other acoustics prediction software. It is concluded that the method can predict most acoustic parameters reliably and provides results as accurate as current commercial room acoustic prediction software. Although the presented acoustic radiance transfer method relies on geometrical acoustics, it can be extended to model diffraction and transmission through materials in future. (ㅇ 2007 Acoustical Society of America.
\end{abstract}

[DOI: $10.1121 / 1.2766781]$

PACS number(s): 43.55.Ka, 43.55.Br, 43.20.Dk [NX]

Pages: $1624-1635$

\section{INTRODUCTION}

There are various methods for geometrical room acoustics modeling, such as the image source, ${ }^{1,2}$ ray tracing, ${ }^{3}$ beam tracing, ${ }^{4}$ and acoustic radiosity ${ }^{5-9}$ methods. Although they have certain similarities, their theoretical foundations are often derived for each method separately. These techniques rely heavily on ray optics and methods borrowed from computer graphics.

In this paper a proper foundation for geometrical room acoustics modeling is introduced. An integral equation, which generalizes a variety of known geometrical room acoustics modeling methods, is constructed. In addition, a novel modeling method, based on the radiosity method, is derived by utilizing the presented theoretical basis. This acoustic radiance transfer solution is shown to be applicable to model sound propagation in realistic, complex environments having surfaces of various materials. A comparison with actual measurement data and data predicted by other acoustics modeling software is given.

In the following, the related work to motivate the proposed room acoustic rendering equation is overviewed. After this, the equation is constructed piece by piece. Next, the widely used geometrical room acoustics modeling methods are shown to be specializations of the proposed general model. The acoustic radiance transfer is introduced with the algorithm description and experimental validation. Finally, possible extensions and future work are discussed.

\section{RELATED WORK}

Computer graphics models the propagation of light in an environment. The speed of light is, in most cases, assumed to be infinite, which is, in practice, a safe assumption since no information can travel faster than light. Sound, however, has

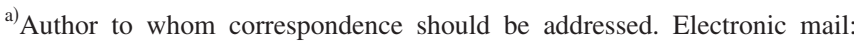
samuel.siltanen@tml.hut.fi
}

to be modeled as waves propagating at finite speed. In room acoustics, the time dependence, due to propagation delays, also has to be modeled. Thus, the room acoustic rendering equation proposed here can be seen as a time-dependent extension of Kajiya's "rendering equation". ${ }^{10}$ The formulation used by Kajiya is rarely seen, and in the modern form the rendering equation can be written as ${ }^{11}$

$$
\begin{gathered}
L(x \rightarrow \Theta)=L_{\mathrm{e}}(x \rightarrow \Theta)+\int_{A} f_{\mathrm{r}}(x, \Psi \rightarrow \Theta) \\
\times L(y \rightarrow-\Psi) V(x, y) G(x, y) \mathrm{d} A_{y},
\end{gathered}
$$

where $L(x \rightarrow \Theta)$ is the outgoing radiance from $x$ in the direction $\Theta . L_{\mathrm{e}}(x \rightarrow \Theta)$ is the emitted radiance from point $x$ in the direction $\Theta, G(x, y)$ is a geometry term, $V(x, y)$ is a visibility term, and $f_{\mathrm{r}}(x, \Psi \rightarrow \Theta)$ is a bidirectional reflectance distribution function. The meaning of the corresponding terms is explained in the context of the time-dependent form presented in the next section.

Since the goal of the presented room acoustics modeling method is to model arbitrary reflections, a flexible representation is required for the reflections. In computer graphics, bidirectional reflectance distribution functions (BRDFs) are widely used to accurately describe the reflections from different types of materials. ${ }^{12}$ Figure 1 shows an example of a BRDF representing nontrivial reflection properties of a material. Combining BRDFs with the radiosity method has been discussed, and the radiosity solution has been extended to handle some nondiffuse reflections in time-independent form. ${ }^{13,14}$ The directional resolution of the solution greatly affects the quality of the final image, and because the memory requirements set serious restrictions on the resolution, these techniques have not been applied to computer graphics to a great extent.

Usually, in sound rendering, either scattering coefficients or diffusion coefficients, in addition to the absorption coefficients, are used to describe the properties of the reflec- 


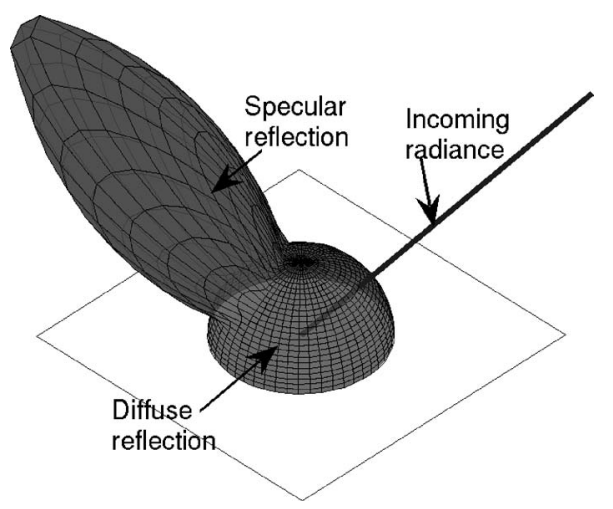

FIG. 1. A BRDF represents the directional distribution of the reflected energy for each incoming angle. Here the distribution for one angle is shown.

tions from acoustic materials. ${ }^{15}$ However, although there is measurement data of the directional distribution of acoustic reflections, the data are paradoxically used to construct random-incident scattering coefficients thus losing the directional information. ${ }^{16}$ There is no clear reason why the complete data of acoustic BRDFs could not be utilized. In this paper BRDFs are applied and it is shown how the BRDF models are constructed from the absorption and scattering coefficients given at each frequency band.

The time-dependent directional extension of the radiosity method is similar to the directional extensions in computer graphics, ${ }^{13,14}$ but because the chosen BRDF representation and the application area are different from the previous work, the extension is derived from the presented theoretical basis. Due to the time dependence, one additional dimension is required, which increases computational requirements. To compensate this, the resolution of the directional data can be reduced since human hearing cannot localize the direction of sounds as accurately as vision can detect the direction of light.

\section{ROOM ACOUSTIC RENDERING EQUATION}

The acoustic energy propagation model presented here models the propagation of acoustic energy in an enclosure. The model is derived by beginning from the reflectance function and concluding with the room acoustic rendering equation. The detection of the acoustic energy by a receiver is presented. Then the issue of frequency dependence is covered, and limitations of the energy-based approach are discussed. The construction of equations is based on a MSc thesis by Kiminki (http://www.niksula.hut.fi/ skiminki/Dskiminki.pdf).

\section{A. Terminology}

In this paper, the terminology established in optics and computer graphics is used, because this way the analogy between those disciplines and room acoustics can be readily utilized. Intensity $I$ is the intensity of energy flux. Irradiance $E(x, \Omega)$ is the incident power on point $x$ on a surface from direction $\Omega$, and radiance $L(x, \Omega)$ is the radiant power per unit projected area per unit solid angle. Symbols $E(x, \Omega, t)$ and $\ell(x, \Omega, t)$ are used for time-dependent irradiance and radiance, respectively, to emphasize the time dependence. For clarity, some of the parameters may be omitted in the construction below.

\section{B. Reflectance distribution function}

The reflectance function describes the directional distribution of the energy arriving at a surface point from a specified incident angle. This can be written in the form of an acoustic BRDF $\rho\left(\Omega_{\mathrm{i}}, \Omega_{\mathrm{e}} ; x^{\prime}\right)$ at point $x^{\prime}$ for incident angle $\Omega_{\mathrm{i}}$ and outgoing angle $\Omega_{\mathrm{e}}$. Analogously to the BRDF in optics, ${ }^{12}$ this six-dimensional function can be defined at point $x^{\prime}$ as the ratio of the differential radiance reflected in an outgoing direction, and the differential irradiance incident through a differential solid angle

$$
\rho\left(\Omega_{\mathrm{i}}, \Omega_{\mathrm{e}} ; x^{\prime}\right)=\frac{\mathrm{d} L\left(\Omega_{\mathrm{e}}\right)}{\mathrm{d} E\left(\Omega_{\mathrm{i}}\right)} .
$$

It is essential to note that the BRDF describes the acoustic material of the surface. By utilizing various BRDFs, modeling different types of reflections is possible. In this paper the BRDFs are assumed time invariant. The BRDF can be written in polar form as $\rho^{\prime \prime}\left(\theta_{\mathrm{i}}, \phi_{\mathrm{i}}, \theta_{\mathrm{e}}, \phi_{\mathrm{e}} ; x^{\prime}\right)$ where the $\theta$ 's are the azimuth angles and the $\phi$ 's are the elevation angles.

\section{Propagation operator}

The operator $\hat{S}_{r}$ represents propagation effects on sound radiation over distance $r$. For an absorptive linear medium it is defined as

$$
\hat{S}_{r} I(t)=e^{-\alpha r} S_{r} I(t)=e^{-\alpha r} I\left(t-\frac{r}{c}\right),
$$

where $c$ is the speed of sound and $\alpha$ is the absorption coefficient. For a nonabsorptive medium, $\alpha=0$, in which case the operator is denoted as $S_{r}$.

It is assumed that the sound is emitted as an impulse at $t=0$ and is denoted by the Dirac delta function $\delta(t)$. Thus, the following properties hold:

$$
\begin{aligned}
& \hat{S}_{r}[\lambda \delta(t)+\mu \delta(t)]=\left[\lambda \hat{S}_{r}+\mu \hat{S}_{r}\right] \delta(t), \\
& \hat{S}_{r_{1}} \hat{S}_{r_{2}} \delta(t)=\hat{S}_{r_{1}+r_{2}} \delta(t), \\
& \hat{S}_{r} \delta(t)=e^{-\alpha t c} S_{r} \delta(t) .
\end{aligned}
$$

Equations (4) and (5) justify combining the propagation operators when all the reflections are specular. The intensity at any point is essentially the sum of intensities transmitted through the direct path and the reflections reaching that point. Further, the linearity property enables differentiation, and, therefore, $S_{r}$ may be used on irradiance and radiance as well.

By using Eq. (6), considering only the response of the impulse energy in the reflection calculation and applying later the effects of air absorption and treatment of an arbitrary source signal is allowed: 


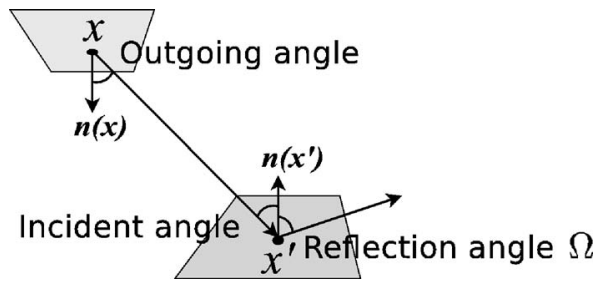

FIG. 2. The geometry term calculation between points $x$ and $x^{\prime}$. Surface normals at these points are $\mathbf{n}(x)$ and $\mathbf{n}\left(x^{\prime}\right)$ correspondingly. Thus the cosine of the incident angle at point $x^{\prime}$ equals $\mathbf{n}\left(x^{\prime}\right) \cdot\left(x-x^{\prime}\right)\left|x-x^{\prime}\right|^{-1}$ and the cosine of the outgoing angle at point $x$ equals $\mathbf{n}(x) \cdot\left(x^{\prime}-x\right)\left|x^{\prime}-x\right|^{-1}$. These factors, in addition to the propagation operator $S_{\left|x-x^{\prime}\right|}$ and the quadratic falloff, determine the geometry term.

$$
I(t)=\left[\sum_{n} \beta_{n} S_{r_{n}} \delta(t)\right] * e^{-\alpha t c} I_{0}(t)=\mathcal{H} \sum_{n} \beta_{n} S_{r_{n}} \delta(t),
$$

where $I_{0}(t)$ is the emission intensity varied over time, $\beta_{n} \mathrm{~s}$ are the reflection coefficients, $r_{n} \mathrm{~s}$ are the lengths of reflection paths, and $I(t)$ is the detected intensity. $\mathcal{H}$ is defined for convenience to incorporate the effects of air absorption and the arbitrary source.

\section{Reflected radiance and geometry term}

A geometry term $g$, similar to that in Eq. (1), is also required in acoustics to describe the effects of the geometry on the acoustic energy flow. It can be derived from the situation depicted in Fig. 2. The differential solid angle $\mathrm{d} \omega_{\Theta}$, in which the differential irradiance is arriving from point $x$ at point $x^{\prime}$, can be expressed using the differential area $\mathrm{d} A_{x}$ in the neighborhood of $x$ as ${ }^{17}$

$$
\mathrm{d} \omega_{\Theta}=\left\lfloor\mathbf{n}(x) \cdot \frac{x^{\prime}-x}{\left|x^{\prime}-x\right|} \mid \frac{\mathrm{d} A_{x}}{\left|x-x^{\prime}\right|^{2}} .\right.
$$

The differential radiance reflected from $x^{\prime}$ to direction $\Omega$ is

$$
\mathrm{d} \ell\left(x^{\prime}, \Omega\right)=\rho\left(\mathrm{d} \omega_{\Theta}, \Omega ; x^{\prime}\right) \mathrm{d} \varepsilon\left(x^{\prime}, \mathrm{d} \omega_{\Theta}\right) .
$$

The differential incident irradiance $\mathrm{d} \varepsilon\left(x^{\prime}, \mathrm{d} \omega_{\Theta}\right)$ may be substituted with $S_{\left|x^{\prime}-x\right|} \ell\left(x,-\mathrm{d} \omega_{\Theta}\right)\left[\mathbf{n}\left(x^{\prime}\right) \cdot\left(x-x^{\prime}\right)\left|x-x^{\prime}\right|^{-1}\right] \mathrm{d} \omega_{\Theta}$, thus yielding

$$
\begin{aligned}
\mathrm{d} \ell\left(x^{\prime}, \Omega\right)= & \rho\left(\mathrm{d} \omega_{\Theta}, \Omega ; x^{\prime}\right) S_{\left|x-x^{\prime}\right|} \ell\left(x,-\mathrm{d} \omega_{\Theta}\right) \\
& \times\left\lfloor\mathbf{n}\left(x^{\prime}\right) \cdot \frac{x-x^{\prime}}{\left|x-x^{\prime}\right|}\left|\mathbf{n}(x) \cdot \frac{x^{\prime}-x}{\left|x^{\prime}-x\right|}\right| \frac{\mathrm{d} A_{x}}{\left|x-x^{\prime}\right|^{2}}\right.
\end{aligned}
$$

By defining the acoustic geometry term as

$$
g\left(x, x^{\prime}\right)=\left\lfloor\mathbf{n}(x) \cdot \frac{x^{\prime}-x}{\left|x^{\prime}-x\right|} \| \mathbf{n}\left(x^{\prime}\right) \cdot \frac{x-x^{\prime}}{\left|x-x^{\prime}\right|} \mid \frac{S_{\left|x-x^{\prime}\right|}}{\left|x-x^{\prime}\right|^{2}},\right.
$$

the differential reflected radiance can be written as

$$
\mathrm{d} \ell\left(x^{\prime}, \Omega\right)=\rho\left(\mathrm{d} \omega_{\Theta}, \Omega ; x^{\prime}\right) g\left(x, x^{\prime}\right) \ell\left(x,-\mathrm{d} \omega_{\Theta}\right) \mathrm{d} A_{x} .
$$

Thus, the acoustic geometry term $g$ is the geometry term used in computer graphics ${ }^{10}$ combined with the propagation operator.

\section{E. Visibility term}

To make the model applicable to nonconvex geometries, it is necessary to include a visibility term. The visibility term $\mathcal{V}\left(x, x^{\prime}\right)$ is defined as follows:

$$
\mathcal{V}\left(x, x^{\prime}\right)= \begin{cases}1, & \text { when the line between } x \text { and } x^{\prime} \\ & \text { is unobstructed } \\ 0, & \text { otherwise. }\end{cases}
$$

\section{F. Reflection kernel}

The reflectance function, the geometry term, and the visibility term together can be used to describe the reflection from point $x$ via point $x^{\prime}$ into direction $\Omega$ (see Fig. 2) as

$$
R\left(x, x^{\prime}, \Omega\right)=\mathcal{V}\left(x, x^{\prime}\right) \rho\left(\frac{x-x^{\prime}}{\left|x-x^{\prime}\right|}, \Omega ; x^{\prime}\right) g\left(x, x^{\prime}\right) .
$$

This is the reflection kernel of the room acoustic rendering equation defined in the next section. Note that the propagation operator $S_{\left|x-x^{\prime}\right|}$ is contained in the acoustic geometry term.

\section{G. Room acoustic rendering equation}

Since the outgoing time-dependent radiance at any point is a combination of the reflected time-dependent radiance, as defined by Eq. (12), and the emitted time-dependent radiance, the acoustic radiance propagation in an enclosure can be written in the form of the room acoustic rendering equation

$$
\ell\left(x^{\prime}, \Omega\right)=\ell_{0}\left(x^{\prime}, \Omega\right)+\int_{\mathcal{G}} R\left(x, x^{\prime}, \Omega\right) \ell\left(x, \frac{x^{\prime}-x}{\left|x^{\prime}-x\right|}\right) \mathrm{d} x,
$$

where $\mathcal{G} \subset \mathrm{R}^{3}$ is the set of all surface points in the enclosure and $\ell\left(x^{\prime}, \Omega\right)$ is the outgoing time-dependent radiance from point $x^{\prime}$ in direction $\Omega$. The equation has the Neumann series solution $^{10}$

$$
\begin{aligned}
& \ell_{n+1}\left(x^{\prime}, \Omega\right)=\int_{\mathcal{G}} R\left(x, x^{\prime}, \Omega\right) \ell_{n}\left(x, \frac{x^{\prime}-x}{\left|x^{\prime}-x\right|}\right) \mathrm{d} x, \\
& \ell\left(x^{\prime}, \Omega\right)=\sum_{n=0}^{\infty} \ell_{n}\left(x^{\prime}, \Omega\right) .
\end{aligned}
$$

In the case of an area source, $\ell_{0}$ represents the radiance emitted by the surface. For point sources, $\ell_{0}$ is considered as the primary reflected radiance instead of defining the source as a part of the surface geometry to make the analysis more convenient. By denoting the position of the source by $x_{\mathrm{s}}$ and the time-dependent intensity of outgoing energy at angle $\Omega$ by $P_{\mathrm{s}}(\Omega, t)$, the time-dependent irradiance from the source to point $x$ can be calculated to be

$$
\varepsilon(x)=\mathcal{V}\left(x, x_{s}\right) \frac{S_{\mid x-x_{s}} P_{s}\left(\frac{x-x_{s}}{\left|x-x_{s}\right|}, t\right)}{4 \pi\left|x-x_{s}\right|^{2}}\left|\mathbf{n}(x) \cdot \frac{x_{s}-x}{\left|x_{s}-x\right|}\right|,
$$

giving the point the primary reflected radiance 


$$
\ell_{0}(x, \Omega)=\rho\left(\frac{x_{s}-x}{\left|x_{s}-x\right|}, \Omega, x\right) \mathcal{V}\left(x_{\mathrm{s}}, x\right) g_{0}\left(x_{s}, x\right) P_{s}\left(\frac{x-x_{s}}{\left|x-x_{s}\right|}, t\right),
$$

where $g_{0}$ is defined as

$$
g_{0}\left(x_{s}, x\right)=\left\lfloor\mathbf{n}(x) \cdot \frac{x_{s}-x}{\left|x_{s}-x\right|} \mid \frac{S_{\left|x_{s}-x\right|}}{4 \pi\left|x_{s}-x\right|^{2}} .\right.
$$

For an omnidirectional point source emitting a unit impulse, the outgoing energy is defined as $P_{s}(\Omega, t)=\delta(t)$.

\section{H. Detection}

For a point receiver, the detection is straightforward. Conceptually, the receiver can be considered as an infinitesimally small sphere, the surface of the sphere detecting only the energy incident from the direction of the surface normal. Additionally, the receiver has a detection transfer function $\Delta(\Omega, I)$ which maps the incident energy arriving from a specific direction to detection, thus taking the possible directional sensitivity pattern into consideration. Since the room acoustic rendering equation models the interaction between surface points only, the direct and indirect sound energy detection have to be handled separately for a receiver that is not part of the geometry. The detection is the sum of the detection of the direct and reflected sound energy

$$
d(t)=d_{\mathrm{D}}(t)+d_{\ell}(t),
$$

where $d_{\mathrm{D}}(t)$ is the detection of the directly detected point source and $d_{\ell}(t)$ is the detection of the reflected radiance

$$
\begin{aligned}
d_{\mathrm{D}}(t)= & \Delta\left(\frac{x_{\mathrm{r}}-x_{s}}{\left|x_{\mathrm{r}}-x_{s}\right|}, \mathcal{H} \mathcal{V}\left(x_{s}, x_{r}\right) \frac{S_{\left|x_{r}-x_{s}\right|}}{4 \pi\left|x_{r}-x_{s}\right|^{2}} P_{s}\left(\frac{x_{r}-x_{s}}{\left|x_{r}-x_{s}\right|}\right)\right), \\
d_{\ell}(t)= & \int_{\mathcal{G}} \Delta\left(\frac{x_{r}-x}{\left|x_{r}-x\right|}, \mathcal{H} \mathcal{V}\left(x, x_{r}\right) \frac{S_{\left|x_{r}-x\right|}}{\left|x_{r}-x\right|^{2}} \ell\left(x, \frac{x_{r}-x}{\left|x_{r}-x\right|}\right)\right. \\
& \left.\times \mid \mathbf{n}(x) \cdot \frac{x_{r}-x}{\left|x_{r}-x\right|}\right) \mathrm{d} x,
\end{aligned}
$$

where $x_{r}$ is the position of the receiver, $x_{s}$ is the position of the point source.

\section{Frequency dependence}

Until now, the frequency dependence in reflections and detection has not been considered. A widely applied technique in room acoustics modeling is to divide the analysis into subbands and consider each subband separately. This technique is valid as long as the subbands can be considered orthogonal, i.e., having no mutual interaction as is the case in typical room acoustics. Thus, the reflections and medium must be linear, as nonlinearity causes harmonics in the signal.

\section{J. Fundamental limitations}

The presented model uses energies instead of pressure disturbances as in the wave field analysis. Therefore, phase information is not included in the model. The energy field approach was chosen since there are difficulties in modeling a general reflection for wave fields. In the case of ideal specular reflections, it is possible to construct a reflection model for a wave field, which has been used in, e.g., the image source method. ${ }^{1}$ However, the task is nontrivial in the case of arbitrary reflections presented as BRDFs.

\section{SPECIALIZATIONS OF THE MODEL}

In this section the different geometrical room acoustics modeling methods are shown to be specializations of the general model given in the previous section. The visibility and acoustic geometry terms remain mostly unchanged while the reflectance function varies from method to method. Most of the commercially available acoustics prediction software are hybrids of the techniques presented below. ${ }^{18,19}$

\section{A. Image source method}

In the basic image source method only the specular reflections are modeled. It can be extended to handle diffraction and scattering, ${ }^{20}$ but, for simplicity, only the basic method is covered in the following discussion. The phase information can be taken into account, although, in practice, the calculations are often done only with energies. For a rectangular room there exists an exact solution. ${ }^{21}$ That solution can also be derived from the presented model by using the Neumann series formulation in Eq. (16). With more complex geometries the solution can be approximated by using the first $n$ terms of the Neumann series, thus modeling the specular reflections up to the $n$th order.

The ideal specular reflectance function used in the image source method is

$$
\rho_{\text {spec }}\left(\Omega_{\mathrm{i}}, \Omega_{\mathrm{e}} ; x\right)=\frac{\beta(x)}{\Omega_{\mathrm{i}} \cdot \mathbf{n}(x)} \delta\left(\Omega_{\mathrm{i}}-M\left(\Omega_{\mathrm{e}}\right)\right),
$$

where $\delta$ is the Dirac delta function, $M$ is the mirror reflection transformation $M(\theta, \phi)=(\theta, \pi-\phi)$, and the reflectivity coefficient $\beta=1-\alpha$ for the absorption coefficient $\alpha$. The inverse of the dot product is added to compensate the Lambertian cosine term included in the geometry term.

The beam tracing method ${ }^{4}$ falls into the same category of acoustics modeling methods because it is used to find specular reflections also. Basically, it is simply an optimization of the visibility function computation, which is the same as in the general case.

\section{B. Ray tracing method}

There are several types of ray tracing methods which can be used in room acoustics modeling. Some methods model only specular reflections. ${ }^{3}$ The reflection kernel is then similar to that of the image source method. One approach to improve the accuracy is to include nonspecular reflections in ray tracing. ${ }^{22}$ This way ray tracing is essentially a numerical solving method for the reflection-iterative construction of the room acoustic rendering equation. A large number of rays is required to accurately model the sound field. Each ray can be thought of as carrying a small portion of the energy emitted 
by the source. The current energies of the rays hitting the receiver are then collected to construct the energy response for the emitted impulse. From this point of view, $\rho$ defines the reflection probability distribution so that a reflection from a ray incident with angle $\Omega_{\mathrm{i}}$ is reflected into direction $\Omega_{\mathrm{e}}$ with probability $\xi\left(\omega_{\mathrm{i}}, \omega_{e}\right)$, which is the biconical reflectance factor

$$
\begin{aligned}
\xi\left(\omega_{\mathrm{i}}, \omega_{\mathrm{e}}\right)= & \left(\int_{\omega_{\mathrm{i}}} \cos \theta_{\mathrm{i}} \mathrm{d} \omega_{\mathrm{i}}\right)^{-1} \\
& \times \int_{\omega_{\mathrm{e}}} \int_{\omega_{\mathrm{i}}} \rho\left(\Omega_{\mathrm{i}}, \Omega_{\mathrm{e}}\right) \cos \theta_{\mathrm{i}} \cos \theta_{\mathrm{e}} \mathrm{d} \omega_{\mathrm{e}} d \omega_{\mathrm{i}},
\end{aligned}
$$

where $\theta_{\mathrm{i}}$ and $\theta_{\mathrm{e}}$ are the elevation angles of $\Omega_{\mathrm{i}}$ and $\Omega_{\mathrm{e}}$, respectively. This evaluates the average reflectance when the incident radiation comes from a set of directions $\omega_{\mathrm{i}}$ and is reflected in a set of directions $\omega_{\mathrm{e}}$. The solid angles covered by the sets of incoming and outgoing radiance are dependent on the implementation. Because integrating $\rho$ over a hemisphere of outgoing angles gives $1-\alpha$ the ray is terminated with probability $\alpha$ thus correctly modeling absorption.

\section{Radiosity method}

The radiosity method can be derived from the room acoustic rendering equation by using the diffuse reflectance function

$$
\rho_{\text {diff }}(x)=\beta(x) / \pi,
$$

where $\beta$ is the reflectivity coefficient of the material at point $x$. This simplifies the room acoustic rendering equation by omitting the directional dependence. Then, discretizing the geometry $\mathcal{G}$ into patches transforms the room acoustic rendering equation into the matrix form discussed in the next section.

\section{Sonel mapping}

Sonel mapping technique ${ }^{23}$ uses the same framework as the photon mapping technique ${ }^{24}$ in computer graphics. The BRDFs are assumed to be combinations of specular and diffuse reflections. A stochastic approach is used where a large number of "sonels" are used for carrying a portion of the acoustic energy sent by the sound source through their reflection paths which consist of ideal specular and diffuse reflections. The reflection paths ending in a diffuse reflection are modeled by the sonel map constructed in the first phase of the algorithm. The specular reflections from the sound source or from the diffuse surfaces are modeled by using distributed ray tracing. It is possible to split the integral in the room acoustics rendering equation in two parts based on whether the last reflection is diffuse or specular, and thus the sonel mapping algorithm can also be seen as a special case of it. Only the diffraction modeling requires special treatment.

\section{ACOUSTIC RADIANCE TRANSFER METHOD}

In this section, the progressive radiosity solution ${ }^{25}$ of the rendering equation in computer graphics is extended to solve the room acoustic rendering equation. In addition, the radi- osity algorithm is further extended to handle arbitrary materials represented by BRDFs, thus extending the radiosity method to model the general acoustic radiance transfer. The reflection models and their construction are discussed in the context of the directional extension.

\section{A. Temporal extension}

The derivation of the theoretical framework for the method can be started with discretizing the geometry $\mathcal{G}$ into a finite number of patches, $N$. The surface of the patch $i$ is denoted by $A_{i}$. Rewriting Eq. (16) using patches yields

$$
\ell_{n+1}\left(x^{\prime}, \Omega\right)=\sum_{i=1}^{N} \int_{A_{i}} R\left(x, x^{\prime}, \Omega\right) \ell_{n}\left(x, \frac{x^{\prime}-x}{\left|x^{\prime}-x\right|}\right) \mathrm{d} x .
$$

By assuming planar patches, the average time-dependent reflected radiance of patch $i$ can be written as

$$
\ell_{n}^{i}(\Omega)=A_{i}^{-1} \int_{A_{i}} \ell_{n}(x, \Omega) \mathrm{d} x
$$

Using this approximation, Eq. (26) can be written as

$$
\ell_{n+1}^{j}(\Omega)=A_{j}^{-1} \sum_{i=1}^{N} \int_{A_{j}}\left[\int_{A_{i}} R\left(x, x^{\prime}, \Omega\right) \ell_{n}^{i}\left(\frac{x^{\prime}-x}{\left|x^{\prime}-x\right|}\right) \mathrm{d} x\right] \mathrm{d} x^{\prime},
$$

where point $x^{\prime}$ is located on patch $j$. The next step is to discretize the reflection kernel. For this, the surface-side hemisphere of a reflection point is divided into a finite number of segments. The segments are denoted as $\vartheta_{j}(k)$. A discretizing projection operator $\hat{\Gamma}_{j}(\Omega)=k$ maps direction $\Omega$ into segment index $k$. Using this discretization, the reflection operator is written as

$$
\hat{R}(i, j, k)=\frac{\int_{A_{i}}\left[\int_{A_{j}}\left[\int_{\vartheta_{j}(k)} R\left(x, x^{\prime}, \Omega\right) \mathrm{d} \Omega\right] \mathrm{d} x^{\prime}\right] \mathrm{d} x}{\int_{A_{i}} \mathrm{~d} x \int_{A_{j}} \mathrm{~d} x^{\prime} \int_{\vartheta_{j}(k)} \mathrm{d} \Omega}
$$

and the emitted radiance as $\hat{\ell}_{n}^{i}(k)$. The discretized reflection operator $\hat{R}$ can be approximated using, e.g., Monte Carlo methods. Using the discretized reflection operator and radiance, Eq. (28) can be written as

$$
\begin{aligned}
\hat{\ell}_{n+1}^{j}(k) & =A_{j}^{-1} \sum_{i=1}^{N} \int_{A_{j}}\left[\int_{A_{i}} \hat{R}(i, j, k) \hat{\ell}_{n}^{i}\left(\hat{\Gamma}_{i}\left(\frac{x^{\prime}-x}{\left|x^{\prime}-x\right|}\right)\right) \mathrm{d} x\right] \mathrm{d} x^{\prime} \\
& =\sum_{i=1}^{N} F_{i, j} \hat{\ell}_{n}^{i} .
\end{aligned}
$$

The operator $F_{i, j}$ is a linear mapping that maps the emitted or reflected energy from patch $i$ into reflected energy from patch $j$ with appropriate time shifting and temporal spreading. If the patches are assumed small compared to the distance between them, temporal spreading may be ignored and the average distance may be used as an approximation for the distance between points in the patches. $F_{i, j}$ may now be represented by a time-shifting operator combined with a dis- 
cretized energy distribution mapping. If $\hat{\ell}_{n}^{j}$ :s are collected into vector $\phi_{n}$ and $F_{i, j}$ : s into matrix $F$,

$$
\phi_{n}=\left(\begin{array}{c}
\hat{\ell}_{n}^{1} \\
\vdots \\
\hat{\ell}_{n}^{N}
\end{array}\right), \quad F=\left(\begin{array}{ccc}
F_{1,1} & \cdots & F_{1, N} \\
\vdots & \ddots & \vdots \\
F_{N, 1} & \cdots & F_{N, N}
\end{array}\right), \quad F_{j, j}=0,
$$

then the $n$th order reflected energy can be mapped into the reflected energy of order $n+1$ as

$$
\phi_{n+1}=F \phi_{n} .
$$

Finally, after discretizing time in $F$ and $\phi$, the acoustic rendering equation is completely discretized for computation

$$
\phi=\sum_{i=0}^{\infty} F^{i} \phi_{0}=\phi_{0}+\sum_{i=1}^{\infty} F^{i} \phi_{0} .
$$

The sum with infinite number of terms in Eq. (33) can be rewritten by utilizing the properties of geometric series

$$
\phi=\phi_{0}+(I-F)^{-1} \phi_{0} \text {. }
$$

If the elements of matrix $F$ contained only real numbers, it could be possible to use standard mathematical methods which are guaranteed to converge, such as Gauss-Seidel or Jacobi methods, for computing the numerical solution. Now, the delay operator in the acoustic geometry term complicates the situation since the elements of matrix $F$ are not real numbers and the elements of vector $\phi$ are time-dependent responses. The delay operator, however, does not prevent utilizing other techniques, such as progressive refinement. ${ }^{25}$ Instead of single energy values, time-dependent energy responses, representing the outgoing energy, are stored for each patch. The responses can be discretized by using an appropriate sampling rate for the convolution required in the detection to be efficient enough. Since the sound energy left in an enclosure asymptotically approaches zero as a function of time, it is practical to cut the tail of the response when the energy is likely to stay below some threshold value.

Equation (30) would yield the accurate form factor $F_{i, j}$, but in practice the analytical solution is too difficult to calculate. To keep the numerical solution simple and efficient, it is assumed that the integrand in Eq. (30) varies very little over patch $i$. Then, the area-to-area form factor can be approximated by the differential-to-finite-area form factor. ${ }^{26}$ The accuracy which is required by the target application and the computational complexity which can be afforded determine the choice of the approach. In this case, Monte Carlo integration is used since it is simple and, in most cases, accurate enough.

\section{B. Directional extension}

The reflectance function $\rho$ can be an arbitrary BRDF. The directional information is discretized using a hemisphere divided into solid angles, as shown in Fig. 5. The BRDFs are thus approximated by the discretized reflectance functions $\hat{\rho}(i, j, k, l ; x)$, where $i$ and $k$ are integers in the range $[0, n$ $-1]$ and $j$ and $l$ are integers in the range $[0, m-1]$ when the hemisphere is divided into $n$ azimuth angles and $m$ elevation angles. The discretized values are computed as integrals over the outgoing directional segment with the average angle of the incoming segment. The results approach the accurate values when the discretization resolutions $n$ and $m$ approach infinity. The discretizations of the BRDFs are precalculated to make the evaluation of the reflection kernel fast at run time.

The patches do not contain only one energy response but $m \times n$ energy responses, one for each solid angle. When a patch receives energy from direction $(i, j)$ the distribution values of the reflectance function $\hat{\rho}(i, j, \cdot, \cdot ; x)$ are scaled by the received energy and added to the directional responses. Thus, only the outgoing energies have to be saved.

Because there is no measured acoustic BRDF data available, the BRDFs can be constructed using the absorption and scattering coefficients given to the materials. The idea is to blend the ideal diffuse BRDF and the ideal specular BRDF

$$
\rho\left(\Omega_{\mathrm{i}}, \Omega_{\mathrm{e}} ; x\right)=(1-s) \rho_{\text {spec }}\left(\Omega_{\mathrm{i}}, \Omega_{\mathrm{e}} ; x\right)+s \rho_{\text {diff }}(x),
$$

where $\rho_{\text {spec }}$ and $\rho_{\text {diff }}$ are as in Eqs. (23) and (25), weighted with the scattering coefficient $s$. When this is discretized as explained above, the specular direction spreads into the whole solid angle. Thus it is called "pseudospecular" reflection in the rest of the paper. Modeling the specular reflection explicitly could be done as in computer graphics, ${ }^{14}$ but this is likely to cause an explosion in the computational requirements since most of the surfaces have the specular component. When using isotropic BRDFs which do not vary over the surface, only three dimensions are needed since the surface coordinates and the incoming azimuth angle can be omitted.

\section{Algorithm description}

The algorithm follows the structure of the progressive radiosity algorithm. ${ }^{25}$ Figure 3 gives the pseudocode of the algorithm. At the main level (see section A in Fig. 3), the algorithm consists of three phases: initial shooting, iterative energy propagation, and final gathering. Figure 4 shows a visual representation of the algorithm in progress.

The first step is to distribute the energy from each sound source to all visible patches. In this case the differential-tofinite-area form factor is accurate if the sources are assumed to be point-like. However, the proposed method easily allows modeling area sound sources with patches emitting sound. In addition, volumetric sound sources can be modeled by handling their bounding surfaces as area sound sources. Sound source directivity can be taken into account at this phase by weighting the outgoing energy flow according to the sound source directivity pattern.

The energy is propagated between the patches until the solution converges, i.e., the total unshot energy drops below a desired threshold value. Assuming that at least one of the patches absorbs energy, the energy will approach zero. In addition, since in the implementation the response length is limited, the speed of sound is finite, and the distances between the patches are nonzero, it follows that after some time no energy is propagated between the patches anymore.

At every iteration, patch $P$ with the highest unshot energy is chosen. This can be done in constant time by using an 


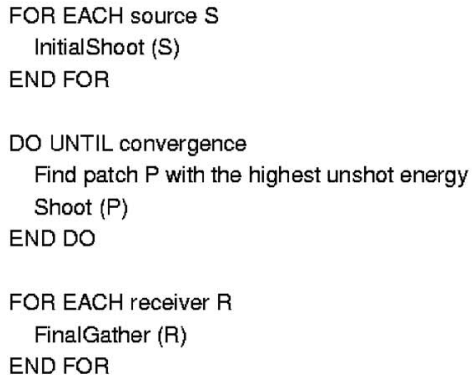

ordered data structure. The unshot energy of patch $P$ is then distributed (see section B in Fig. 3). If there exists previously computed data for that patch they can be used directly. Otherwise, each patch $Q$, that is even partially visible to patch $P$, is sampled uniformly for the Monte Carlo integration (see section $\mathrm{C}$ in Fig. 3). The weighted form factors and propagation delays from the centroid of patch $P$ to each sample on patch $Q$ are accumulated and normalized to get the final results. During this process the information about the discretized outgoing angles at patch $P$ and the incoming angles at patch $Q$ is computed and stored into a structure indexed by the outgoing angle, target patch, and the incoming angle. Then, it is straightforward to go through each outgoing direction at patch $P$ and find the corresponding incident direction for each outgoing direction at patch $Q$, and weight the form factors according to the outgoing solid angle and the proportion of it covered by the incident solid angle at the receiving patch (see section $\mathrm{D}$ in Fig. 3). The energy responses are then combined, which is the most timeconsuming part of the algorithm when the responses consist of thousands of samples. To better simulate high frequencies, air absorption is introduced. ${ }^{27}$ It is embedded in the calculation by weighting the accumulated responses according to the absorption factors for the distance between the patches.

The final gathering phase can be seen as the opposite of the initial shooting. The energy responses from all visible patches are accumulated for each listener. The Monte Carlo integration is different, however. The form factor and propagation delay values are not accumulated and normalized, but used in convolving the responses from individual samples directly. The directional information is thus better preserved in the accumulated response. In addition, the directional information can be saved explicitly, or it can be used to weight the incoming energy to emulate microphones with different directivities or head related transfer functions.

The number of sound sources affects only the complexity of the initial shooting. Since the initial shooting is a fast operation, modeling multiple sound sources with the same emitted signal at once takes practically the same total time as modeling only one sound source.

It is worth noting that the solution stored in patches is view independent, and thus only the final gathering operation 

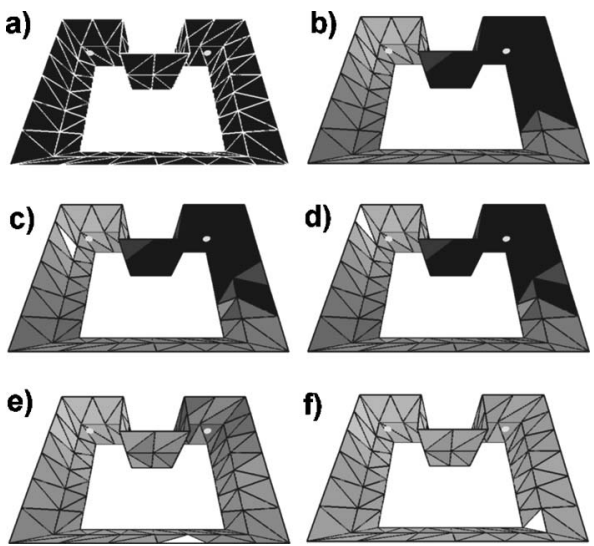

FIG. 4. The progressive radiance transfer solution. (a) The model without floor and ceiling before the initial shooting, where the source is the light dot on the left and the receiver is the dot on the right. (b) The model after the initial shooting, where the lightness of the patch is proportional to the amount of energy it has received. (c) The patch with the highest energy is emphasized, and the energy reflected from it has been added to the patches visible to that patch. This patch now no longer has the highest energy. (d) The next patch with the highest unshot energy is shown, and the energy is propagated similarly to (d). (e) The situation several steps later, and (f) the energy distribution in the model when the solution has converged. The energy from patches visible to the receiver is collected into it in the final gathering phase.

is required for different receiver positions. Since the final gathering operation takes only a few milliseconds in typical models, the receiver position can be changed in real time. When combined with an efficient audio renderer, this solution would allow interactive walk-through auralizations in scenes with stationary sound sources and geometry.

\section{RESULTS}

The algorithm is evaluated with the geometry given in the third round robin test on room acoustical computer simulation by Bork. ${ }^{28,29}$ This geometry is chosen because it is a model of a room for which there is available public acoustic measurement data, in addition to the data produced by other room acoustics prediction software. Three levels of detail for the same model are given. Phase 2 geometry, consisting of 378 patches, is chosen for evaluating the acoustic parameters. It has enough details to produce unique responses at different receiver and source positions, but, on the other hand, the diffusing surfaces are not modeled too accurately to demonstrate the effects of both highly diffuse and highly specular BRDFs. The simulations are run separately for each frequency band with curtains open and closed. In addition, some results for the phase 3 geometry, consisting of 986 patches, are given to assess the algorithm performance with a higher number of patches.

\section{A. Materials}

The materials are given as absorption and scattering coefficients for six octave bands from $125 \mathrm{~Hz}$ to $4 \mathrm{kHz}$. The BRDFs, with a $30^{\circ}$ elevation and azimuth angle resolution, are constructed as explained in the previous section. Figure 5 shows a discretized BRDF for one incident angle.

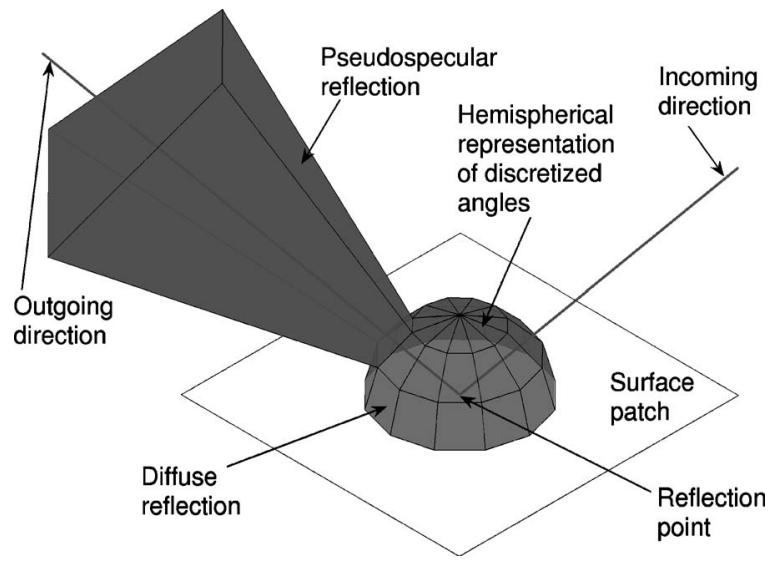

FIG. 5. Hemisphere is divided into solid angles to discretize the directional information. Here, both the azimuth angle resolution and the elevation angle resolution are $30^{\circ}$ yielding 36 directional segments. Thus, the incoming and outgoing directions of the reflections, defined by an azimuth angle $\theta$ and an elevation angle $\phi$, can be mapped onto a pair of corresponding integer values $i$ and $j$. Constructed BRDFs are weighted sums of specular and diffuse reflections. The discretized distribution of the reflected energy for one incident angle is shown. Compare this to the undiscretized BRDF in Fig. 1.

\section{B. Acoustic parameters}

Eight acoustic parameters are compared, namely reverberation time $\left(T_{30}\right)$, early decay time $(\mathrm{EDT})$, definition $\left(D_{50}\right)$, clarity $\left(C_{80}\right)$, center time (TS), strength $(G)$, and lateral energy fractions (LF and LFC). These parameters give good insight into the advantages and disadvantages of the suggested method. The energy responses, from which the parameters are to be extracted, are simulated for two source and three receiver positions and for six octave bands with curtains open and closed. The data in the figures correspond to that shown by Bork for the other room acoustics prediction software. ${ }^{29}$ To emphasize the positional dependency, the Tables I-III contain average values of the four middle frequencies with curtains open for varying positions.

In general, the estimates of $T_{30}$ are very good. The values averaged over four middle octave bands remain within $0.04 \mathrm{~s}$ of the measured values. Figure 6(a) shows the values for each octave band at position S2R2. The values seem to be higher at low frequencies and lower at higher frequencies than the measured data with open curtains. However, the

TABLE I. The measured and simulated $T_{30}$ and EDT values averaged for the $250 \mathrm{~Hz}, 500 \mathrm{~Hz}, 1 \mathrm{kHz}$, and $2 \mathrm{kHz}$ octave bands. Each of the six combinations of source and receiver positions are given.

\begin{tabular}{|c|c|c|c|c|c|c|c|}
\hline \multirow[b]{2}{*}{$\mathrm{Src}$} & \multirow[b]{2}{*}{ Rcv } & \multicolumn{3}{|c|}{$T_{30}$} & \multicolumn{3}{|c|}{ EDT } \\
\hline & & $\begin{array}{c}\text { Meas. } \\
\text { (s) }\end{array}$ & $\begin{array}{c}\text { Sim. } \\
(\mathrm{s})\end{array}$ & $\begin{array}{l}\text { Err. } \\
(\%)\end{array}$ & $\begin{array}{c}\text { Meas. } \\
\text { (s) }\end{array}$ & $\begin{array}{c}\text { Sim. } \\
(\mathrm{s})\end{array}$ & $\begin{array}{l}\text { Err. } \\
(\%)\end{array}$ \\
\hline S1 & $\mathrm{R} 1$ & 1.12 & 1.11 & -0.9 & 1.16 & 1.15 & -1.4 \\
\hline S1 & R2 & 1.15 & 1.11 & -2.8 & 1.07 & 1.08 & 1,8 \\
\hline $\mathrm{S} 1$ & R3 & 1.13 & 1.11 & -1.4 & 1.21 & 1.19 & -1.4 \\
\hline S2 & $\mathrm{R} 1$ & 1.13 & 1.11 & -1.2 & 1.11 & 1.07 & -2.8 \\
\hline $\mathrm{S} 2$ & $\mathrm{R} 2$ & 1.13 & 1.11 & -1.6 & 1.12 & 1.19 & 5.6 \\
\hline $\mathrm{S} 2$ & R3 & 1.11 & 1.11 & 0.4 & 1.11 & 1.10 & -0.5 \\
\hline
\end{tabular}


TABLE II. The measured and simulated $D_{50}, C_{80}$, and TS values averaged for the $250 \mathrm{~Hz}, 500 \mathrm{~Hz}, 1 \mathrm{kHz}$, and $2 \mathrm{kHz}$ octave bands. Each of the six combinations of source and receiver positions are given.

\begin{tabular}{|c|c|c|c|c|c|c|c|c|c|c|}
\hline $\mathrm{S}$ & $\mathrm{R}$ & $\begin{array}{c}\text { Meas. } \\
(\%)\end{array}$ & $\begin{array}{l}D_{50} \\
\text { Sim. } \\
(\%)\end{array}$ & $\begin{array}{l}\text { Err. } \\
(\%)\end{array}$ & $\begin{array}{c}\text { Meas. } \\
(\mathrm{dB})\end{array}$ & $\begin{array}{c}C_{80} \\
\text { Sim. } \\
(\mathrm{dB})\end{array}$ & $\begin{array}{l}\text { Err. } \\
\text { (dB) }\end{array}$ & $\begin{array}{c}\text { Meas. } \\
(\mathrm{ms})\end{array}$ & $\begin{array}{l}\text { TS } \\
\text { Sim. } \\
(\mathrm{ms})\end{array}$ & $\begin{array}{l}\text { Err. } \\
(\%)\end{array}$ \\
\hline $\mathrm{S} 1$ & $\mathrm{R} 1$ & 49.19 & 48.94 & -0.5 & 2.85 & 2.59 & -0.26 & 78.79 & 83.12 & 5.5 \\
\hline $\mathrm{S} 1$ & $\mathrm{R} 2$ & 56.61 & 54.68 & -3.4 & 3.51 & 3.55 & 0.04 & 68.19 & 72.73 & 6.7 \\
\hline $\mathrm{S} 1$ & R3 & 46.13 & 45.04 & -2.4 & 1.90 & 2.16 & 0.26 & 84.52 & 90.56 & 7.2 \\
\hline $\mathrm{S} 2$ & $\mathrm{R} 1$ & 53.87 & 55.53 & 3.1 & 3.09 & 3.51 & 0.41 & 71.25 & 72.72 & 2.1 \\
\hline $\mathrm{S} 2$ & $\mathrm{R} 2$ & 43.49 & 44.33 & 2.0 & 1.94 & 2.20 & 0.25 & 82.69 & 90.81 & 9.8 \\
\hline $\mathrm{S} 2$ & R3 & 53.23 & 51.22 & -2.0 & 3.08 & 3.10 & 0.02 & 71.68 & 77.02 & 7.4 \\
\hline
\end{tabular}

shape of the curve is similar to those shown for other prediction software. ${ }^{29}$ Averaged $T_{30}$ values for each listener and source positions are given in Table I.

The EDT values, presented in Table I, also follow the measured values quite accurately. The averaged values do not differ more than $0.07 \mathrm{~s}$ in the worst case, and these errors can also be partly explained by errors in the room model. However, the variation in individual values is larger than in $T_{30}$. Figure 6(b) shows the values for each position in the $1 \mathrm{kHz}$ octave band. Although there are some deviations from the measured data, the positional dependency in the EDT can be seen clearly. In contrast, according to Bork some room acoustics prediction programs have difficulties in modeling it properly. ${ }^{29}$

The $D_{50}$ values with curtains open are mostly near to the measured data, see Table II. Results are slightly more inaccurate with closed curtains. This can be seen in Fig. 6(c) where the $D_{50}$ values in the $1 \mathrm{kHz}$ octave band for each position are shown. In the lowest octave band $(125 \mathrm{~Hz})$, shown in Fig. 6(d), there are noticeable differences. However, compared to the values given by the other room acoustics prediction software, ${ }^{29}$ the results are good. The $C_{80}$ values show similar behavior, although the relative errors are larger. Figure 6(e) shows the $C_{80}$ values at position S1R1 for each octave band. Since these parameters depend on the direct sound and the early reflections, based on these results it is reasonable to believe the energy of the early part of the response is properly constructed.

The TS values shown in Table II are within the subjective error difference limen $( \pm 10 \mathrm{~ms}),{ }^{28}$ although the simulated values are a little higher than the measured values in general. Because the integral evaluated in calculating the TS values is over the whole response, the late reflections are also taken into account. Thus it can be concluded that the late reflections are modeled properly by the acoustic radiance transfer method. Figure 6(f) shows the values for each octave band at position S1R1.

The simulated LF values are somewhat lower than the measured values while the LFC values are closer to the measured values. Otherwise, their behavior is similar. Figure 6(g) shows the LF values for each octave band at position S1R1. The simulated values do not vary much in relation to the frequency, unlike the measured values. The same phenomenon can be seen in the data produced by the other room acoustics prediction software, ${ }^{29}$ which might indicate that the room model does not properly capture some aspects of the real room. On the other hand, since these parameters consider the directional distribution of the early reflections, especially the LF values are prone to small changes in their incident direction at some locations. It has been shown that the prediction of parameters such as LF and $C_{80}$ are sensitive to the diffusion modeling scheme used, ${ }^{30}$ which could explain some of the errors. The results for both LF and LFC averaged over four middle octave bands with open curtains can be seen in Table III. Figure 6(h) shows the LF values for each position in the $1 \mathrm{kHz}$ octave band. Although the simulated values are lower than the measured values, the positional dependence can be seen.

The simulated $G$ values do not differ much from the measured values. The local dependence, characterized by the direct sound and early reflections, is strong, as can be seen in the measured values in Table III. The behavior of the simulated values is similar in relation to the position of the sound sources and receivers. Figure 6(i) shows $G$ values for each position in the $1 \mathrm{kHz}$ octave band.

Most parameter values extracted from the simulated data

TABLE III. The measured and simulated LF, LFC, and $G$ values averaged for the $250 \mathrm{~Hz}, 500 \mathrm{~Hz}, 1 \mathrm{kHz}$, and $2 \mathrm{kHz}$ octave bands. Each of the six combinations of source and receiver positions are given.

\begin{tabular}{|c|c|c|c|c|c|c|c|c|c|c|}
\hline \multirow[b]{2}{*}{ S } & \multirow[b]{2}{*}{$\mathrm{R}$} & \multicolumn{3}{|c|}{$\mathrm{LF}$} & \multicolumn{3}{|c|}{ LFC } & \multicolumn{3}{|c|}{$\mathrm{G}$} \\
\hline & & $\begin{array}{c}\text { Meas. } \\
(\%)\end{array}$ & $\begin{array}{l}\text { Sim. } \\
(\%)\end{array}$ & $\begin{array}{l}\text { Err. } \\
(\%)\end{array}$ & $\begin{array}{c}\text { Meas. } \\
(\%)\end{array}$ & $\begin{array}{l}\text { Sim. } \\
(\%)\end{array}$ & $\begin{array}{l}\text { Err. } \\
(\%)\end{array}$ & $\begin{array}{l}\text { Meas. } \\
(\mathrm{dB})\end{array}$ & $\begin{array}{l}\text { Sim. } \\
\text { (dB) }\end{array}$ & $\begin{array}{l}\text { Err. } \\
(\%)\end{array}$ \\
\hline S1 & $\mathrm{R} 1$ & 25.55 & 20.21 & -20.9 & 32.31 & 31.18 & -3.5 & 19.33 & 18.86 & -2.5 \\
\hline S1 & $\mathrm{R} 2$ & 20.52 & 18.77 & -8.5 & 28.07 & 28.25 & 0.7 & 20.48 & 20.06 & -2.5 \\
\hline S1 & R3 & 26.86 & 19.53 & -27.3 & 32.00 & 32.49 & 1.6 & 18.94 & 18.72 & -1.2 \\
\hline $\mathrm{S} 2$ & $\mathrm{R} 1$ & 21.00 & 16.69 & -20.5 & 26.33 & 27.61 & 4.9 & 20.01 & 20.91 & 4.5 \\
\hline $\mathrm{S} 2$ & $\mathrm{R} 2$ & 24.61 & 20.41 & -17.1 & 30.11 & 32.55 & 8.1 & 19.36 & 20.31 & 4.9 \\
\hline $\mathrm{S} 2$ & $\mathrm{R} 3$ & 21.92 & 18.25 & -16.7 & 27.88 & 28.55 & 2.4 & 19.98 & 20.73 & 3.8 \\
\hline
\end{tabular}



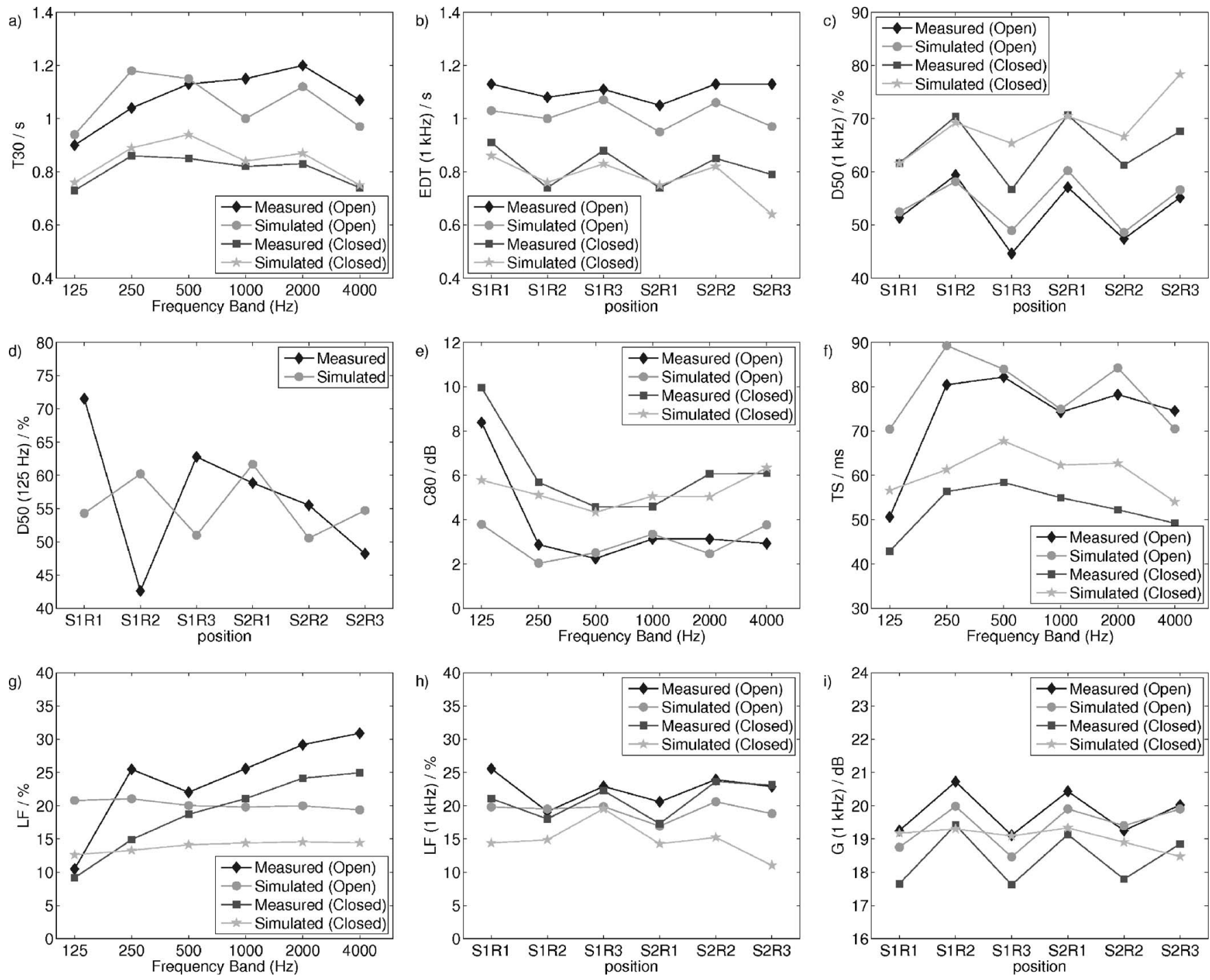

FIG. 6. The charts show (a) $T_{30}$ values at position S2R2 (cf. Fig. 6 in Ref. 30), (b) EDT values in the $1 \mathrm{kHz}$ octave band (cf. Fig. 8 in Ref. 30 ), (c) $D_{50}$ values in the $1 \mathrm{kHz}$ and (d) $125 \mathrm{~Hz}$ octave bands (cf. Figs. 10 and 11 in Ref. 30 respectively), (e) $C_{80}$, (f) TS, and (g) LF values at position S1R1 (cf. Figs. 13, 14, and 19 in Ref. 30, respectively), and (h) LF and (i) G in the $1 \mathrm{kHz}$ octave band (cf. Figs. 20 and 17 in Ref. 30, respectively).

are close to the measured values. In some cases, the computer model of the room sets limitations on the accuracy of the results since the geometry is simplified and some of the material data is only approximate. ${ }^{29}$ Comparing the results produced by the acoustic radiance transfer method to the results of the other acoustics prediction software with the same room model, the frequency dependent behavior of the parameters is very similar, although the results differ from the measured data. This can be explained by both the errors in the room model and the common limitations of the geometrical room acoustics modeling methods. Errors at the lower frequencies can be expected because the wave effects are not properly taken into account. ${ }^{28}$

\section{Computational performance}

The memory consumption of the algorithm is high, in typical cases hundreds of megabytes, because each patch has an energy response for each discretized hemisphere direction. The amount of memory required is directly proportional to the number of patches, number of directional segments, sampling frequency, and the length of the energy response.

The computation times are also quite long with high spatial and temporal resolutions. In addition to the factors mentioned above, the Monte Carlo sampling density and the convergence threshold value also affect the computation time. Table IV contains some computation times on a PC with a Pentium $42.8 \mathrm{GHz}$ processor and $1024 \mathrm{MB}$ random access memory. It is obvious that the running times of the unoptimized version of the acoustic radiance transfer method are longer than with commercial acoustics prediction software, but they are still feasible for practical applications and research purposes.

\section{POSSIBLE EXTENSIONS AND FUTURE WORK}

There are several possible error sources in the acoustic radiance transfer method. Wave effects are not modeled since there is no phase information when calculating with energies. For example, the edge diffraction is not taken into account in 
TABLE IV. Computation times for each octave band with phase 1, phase 2, and phase 3 geometries consisting of 90, 378, and 986 patches, respectively, using angular resolution of $30^{\circ}$ for both azimuth and elevation angles, and a time resolution of $1 \mathrm{~ms}$. The length of the response is $1.0 \mathrm{~s}$, and the energy level for convergence is set to $10^{-4}$ of the initial energy which corresponds to $40 \mathrm{~dB}$ attenuation. Double precision is used for floating points.

\begin{tabular}{lccc}
\hline \hline Octave Band & Phase 1 & Phase 2 & Phase 3 \\
\hline $125 \mathrm{~Hz}$ & $5 \min 33 \mathrm{~s}$ & $24 \min 42 \mathrm{~s}$ & $1 \mathrm{~h} 41 \min 43 \mathrm{~s}$ \\
$250 \mathrm{~Hz}$ & $5 \min 28 \mathrm{~s}$ & $31 \min 13 \mathrm{~s}$ & $2 \mathrm{~h} 13 \min 5 \mathrm{~s}$ \\
$500 \mathrm{~Hz}$ & $5 \min 28 \mathrm{~s}$ & $28 \min 57 \mathrm{~s}$ & $2 \mathrm{~h} 26 \min 25 \mathrm{~s}$ \\
$1 \mathrm{kHz}$ & $5 \min 27 \mathrm{~s}$ & $24 \min 7 \mathrm{~s}$ & $2 \mathrm{~h} 10 \min 25 \mathrm{~s}$ \\
$2 \mathrm{kHz}$ & $5 \min 14 \mathrm{~s}$ & $27 \min 32 \mathrm{~s}$ & $2 \mathrm{~h} 26 \min 56 \mathrm{~s}$ \\
$4 \mathrm{kHz}$ & $4 \min 36 \mathrm{~s}$ & $23 \min 50 \mathrm{~s}$ & $2 \mathrm{~h} 7 \min 58 \mathrm{~s}$ \\
\hline \hline
\end{tabular}

the implementation. The acoustic energy propagation model could be extended to use diffraction models ${ }^{31}$ by modifying only the reflection kernel.

The reflection model could be improved. The relationship of the directional description to scattering and diffusion coefficients should be studied more carefully to assure their proper use. ${ }^{15}$ The discretization of the BRDFs could be replaced by the use of spherical harmonics ${ }^{14}$ so that in the energy response, each sample would consist of spherical harmonics coefficients. In addition, the material representation could possibly be extended to cover nonlocally reacting surfaces by applying bidirectional subsurface radiance distribution functions ${ }^{12}$ which also model subsurface scattering. This would allow transmission through materials, although one temporal and two spatial dimensions must be added to the reflectance model.

\section{CONCLUSIONS}

A model for acoustic energy propagation is presented in the form of the room acoustic rendering equation. Several widely used geometrical room acoustics modeling methods are shown to be specializations of this model. It provides foundations for even more general methods for modeling the propagation of acoustic energy. Thus, a more accurate prediction of room acoustics is made possible.

A new method is proposed to solve the room acoustic rendering equation. The method, called acoustic radiance transfer method, is an extension of the previous work on the acoustic radiosity method, because the time-dependent acoustic radiance transfer method can handle arbitrary reflections, represented by acoustic BRDFs, in addition to the diffuse reflections. The simulated results indicate that the acoustic radiance transfer method is at least as reliable a method for room acoustics prediction as most of the methods used today. In addition, it offers flexibility in modeling arbitrary reflections and handling complex sound sources. This allows the method to be extended to handle diffracting edges and transmission through materials in the future.

\section{ACKNOWLEDGMENTS}

The authors would like to thank D.Sc. Janne Kontkanen for insightful comments on the paper, and Professor Kar- jalainen, Professor Sulonen, and Professor Takala for the early discussions on the subject. This research has been financially supported by the EU's FP6 IST project Uni-Verse.

${ }^{1}$ J. B. Allen and D. A. Berkley, "Image method for efficiently simulating small-room acoustics," J. Acoust. Soc. Am. 65, 943-950 (1979).

${ }^{2}$ J. Borish, "Extension of the image model to arbitrary polyhedra," J. Acoust. Soc. Am. 75, 1827-1836 (1984).

${ }^{3}$ A. Krokstad, S. Strom, and S. Sorsdal, "Calculating the acoustical room response by the use of a ray tracing technique," J. Sound Vib. 8, 118-125 (1968).

${ }^{4}$ T. Funkhouser, N. Tsingos, I. Carlbom, G. Elko, M. Sondhi, J. West, G. Pingali, P. Min, and A. Ngan, "A beam tracking method for interactive architectural acoustics," J. Acoust. Soc. Am. 115, 739-756 (2004).

${ }^{5}$ M. Hodgson and E.-M. Nosal, "Experimental evaluation of radiosity for room sound-field prediction," J. Acoust. Soc. Am. 120, 808-819 (1996).

${ }^{6}$ E.-M. Nosal, M. Hodgson, and I. Ashdown, "Investigation of the validity of radiosity for sound-field prediction in cubic rooms," J. Acoust. Soc. Am. 116, 3505-3514 (2004).

${ }^{7}$ E.-M. Nosal, M. Hodgson, and I. Ashdown, "Improved algorithms and methods for room sound-field prediction by acoustical radiosity in arbitrary polyhedral rooms," J. Acoust. Soc. Am. 116, 970-980 (2004).

${ }^{8}$ T. Lewers, "A combined beam tracing and radiant exchange computer model of room acoustics," Appl. Acoust. 38, 161-178 (1993).

${ }^{9}$ N. Tsingos and J.-D. Gascuel, "Acoustic simulation using hierarchical time-varying radiant exchanges," URL citeseer.ist.psu.edu/721526.html, [viewed 2007-06-27].

${ }^{10}$ J. T. Kajiya, "The rendering equation," Comput. Graph. 20, 143-150 (1986).

${ }^{11}$ P. Dutré, P. Bekaert, and K. Bala, Advanced Global Illumination (A. K. Peters, Wellesley, MA, 2003).

${ }^{12}$ F. E. Nicodemus, J. C. Richmond, J. J. Hsia, I. W. Gingberg, and T. Limperis, "Geometric considerations and nomenclature for reflectance," Monograph 161, National Bureau of Standards (U.S.) (1977).

${ }^{13}$ D. S. Immel, M. F. Cohen, and D. P. Greenberg, "A radiosity solution for non-diffuse environments," Comput. Graph. 20, 133-142 (1986).

${ }^{14}$ F. X. Sillion, J. R. Arvo, S. H. Westin, and D. P. Greenberg, "A global illumination solution for general reflectance distributions," Comput. Graph. 25, 187-196 (1991).

${ }^{15}$ T. J. Cox, B.-I. L. Dalenback, P. D’Antonio, J. J. Embrechts, J. Y. Jeon, E. Mommertz, and M. Vorländer, "A tutorial on scattering and diffusion coefficients for room acoustic surfaces," Acta. Acust. Acust. 92, 1-15 (2006).

${ }^{16} \mathrm{M}$. Vorländer and E. Mommertz, "Definition and measurement of randomincident scattering coefficients," Appl. Acoust. 60, 187-199 (2000).

Here, the following notation is used: $\lfloor a\rfloor=\left\{\begin{array}{l}a, a \geqslant 0 \\ 0, a<0\end{array}\right.$.

${ }^{18}$ G. M. Naylor, "ODEON-another hybrid room acoustical model," Appl. Acoust. 38, 131-143 (1993).

${ }^{19}$ B.-I. Dalenbäck, "Room acoustic prediction based on a unified treatment of diffuse and specular reflection," J. Acoust. Soc. Am. 100, 899-909 (1996).

${ }^{20}$ U. Svensson and U. Kristiansen, "Computational modeling and simulation of acoustic spaces," in AES 22nd International Conference on Virtual, Synthetic and Entertainment Audio, 11-30 (Espoo, Finland) (2002).

${ }^{21}$ H. Kuttruff, Room Acoustics, 4th ed. (Spon, London, 2000).

${ }^{22}$ H. Lehnert and J. Blauert, "Principles of binaural room simulation," Appl. Acoust. 36, 259-291 (1992).

${ }^{23}$ B. Kapralos, "The sonel mapping acoustical modeling method," Ph.D. thesis, York University, Toronto, Ontario, Canada (2006).

${ }^{24} \mathrm{H}$. W. Jensen, Realistic Image Synthesis Using Photon Mapping (A. K. Peters, Natick, MA, 2001).

${ }^{25}$ M. F. Cohen, S. E. Chen, J. R. Wallace, and D. P. Greenberg, "A progressive refinement approach to fast radiosity image creation," Comput. Graph. 22, 75-84 (1988).

${ }^{26}$ M. F. Cohen and D. P. Greenberg, "A radiosity solution for complex environments," Comput. Graph. 19, 31-40 (1985).

${ }^{27}$ H. Bass and H.-J. Bauer, "Atmospheric absorption of sound: Analytical expressions," J. Acoust. Soc. Am. 52, 821-825 (1972).

${ }^{28}$ I. Bork, "Report on the 3rd round robin on room acoustical computer simulation - Part I: Measurements," Acta. Acust. Acust. 91, 740-752 (2005).

${ }^{29}$ I. Bork, "Report on the 3rd round robin on room acoustical computer 
simulation - Part II: Calculations," Acta. Acust. Acust. 91, 753-763 (2005).

${ }^{30}$ Y. W. Lam, “A comparison of three diffuse reflection modeling methods used in room acoustics computer models," J. Acoust. Soc. Am. 100, 2181-
2192 (1996).

${ }^{31}$ U. P. Svensson, R. I. Fred, and J. Vanderkooy, "Analytic secondary source model of edge diffraction impulse responses," J. Acoust. Soc. Am. 106, 2331-2344 (1999). 